\title{
Sharing "Negative" Results in Neuromuscular Research: A Positive Experience
}

\author{
Virginia Arechavala-Gomeza ${ }^{\mathrm{a}, \mathrm{b}, *}$ and Annemieke Aartsma-Rus ${ }^{\mathrm{c}}$ \\ ${ }^{a}$ Neuromuscular Disorders, Biocruces Bizkaia Health Research Institute, Barakaldo, Spain \\ ${ }^{\mathrm{b}}$ Ikerbasque, Basque Foundation for Science, Bilbao, Spain \\ ${ }^{\mathrm{c}}$ Department of Human Genetics, Leiden University Medical Center, ZA, Leiden, The Netherlands
}

Pre-press 20 August 2021

Last year we announced the launch of a special section in this journal - "the null hypothesis stands" - dedicated to studies where results were not those anticipated or hoped for. This special issue fully dedicated to such studies is the formal inauguration of this section. When we announced the section, we were wondering what we may receive. We had recently completed a survey amongst members of our own network of antisense oligonucleotide therapy researchers on sharing such results. This revealed that researchers think results that do not disprove the null hypothesis or that cannot replicate published work are worth sharing. However, at the same time, many think that doing so themselves is a waste of precious time and only a few of the respondents had actually published such work [1]. As such, we had some trepidation that researchers in the neuromuscular field might feel hesitancy towards publishing on studies that disproved the null hypothesis.

It is clear that results that prove an original hypothesis to be incorrect advance knowledge as much as those that confirm it. Still, in the era of "publish or perish" results that disprove the hypothesis tested are

\footnotetext{
*Correspondence to: Prof. Virginia Arechavala-Gomeza, Biocruces Bizkaia Health Research Institute, Barakaldo, Bizkaia, 48903, Spain. Tel.: +34 946007967; E-mail: virginia.arechava lagomeza@osakidetza.eus.; ORCID: 0000-0001-7703-3255
}

not published at the rate that should be expected [2-4], and the lack of reproducibility has also been recently highlighted as a problem that needs to be addressed. A publication bias towards therapeutic approaches that appear to have beneficial effects and lack of published studies where therapies are either not useful or toxic, or where published results are not reproducible [5, 6], not only impedes science and therapy development, but it also leads to unnecessary duplication of efforts and a waste of resources. In any field this is not optimal, but in a rare disease field such as research in neuromuscular disorders this is a tragedy.

In light of this, we are happy to present a whole issue dedicated to well-executed research that obtained what many may consider "negative" results. Included in this special issue, readers may find 8 research articles. They all have been reviewed by peers and present the markings of high-quality research. They include a variety of subjects, from animal models to biomarkers and clinical trials.

We highlight a few examples to illustrate the usefulness of sharing these results. One study assessed whether facio-scapulo-humeral muscular dystrophy (FSHD) patients had auto-antibodies int their serum due to DUX4 activation [7]. After evaluating 138 patients and 20 controls, the answer to this question seems to be negative, but the result nonetheless provides the neuromuscular community with new 
insights. The publication of this manuscript will not only avoid future research duplications, but also the possibility of completing this work with other approaches, such as evaluating a cellular response.

Another study evaluated whether there was an association between HLA class II alleles and disease severity in 146 Iranian patients with myasthenia gravis, an autoimmune NMD [8]. Patients in the cohort had been followed up for 5 years or more. No association was found between the HLA class II haplotype and disease severity or treatment response. As the groups per haplotype were relatively small, due to the inherent variability of HLA genes, it is still possible that an association exists, but one that is small and that can only be picked up in larger groups of patients. Future studies may shed more light on this.

It is known that in mouse models the genetic background can influence the disease severity. For example the $m d x$ mouse model lacking dystrophin has a more severe pathology on a DBA background than on a C57/B110 background, due to amongst others a polymorphism in latent TGF-beta binding protein 4 (Ltbp4) gene that leads to increased deposition of fibrotic tissue. Similarly, the $m d x$ phenotype can be exacerbated by knocking out the cytidine monophosphate sialic adic hydroxylase ( $\mathrm{Cmah}$ ) gene, which is present in mice but not in humans. This difference has been proposed as one of the reasons why generally muscle pathology is less severe in murine knockouts than in neuromuscular disease patients. In a study in this issue authors aimed to assess whether crossing a mouse model with a fukutin related protein (Fkrp) mutation into the DBA background or into a $\mathrm{Cmah}$ mutated background could exacerbate the phenotype of this model [9]. Authors confirmed that these crosses exacerbated the pathology for the $m d x$ mutation, but had no impact on pathology for the Fkrp mutation. While this is disappointing, the fact that the authors share these results mean that others do not have to do the same painstaking task of crossing these animal models to discover that this does not exacerbate the phenotype.

Finally, we would like to highlight the report of the phase 3 clinical trial of edasalonexent treatment in Duchenne patients [10]. Edasalonexent is a potent inhibitor of $\mathrm{NF} \kappa \mathrm{B}$, one of the key proteins driving the inflammatory response that is an important pathological pathway in Duchenne skeletal muscle. Patients are treated with glucocorticosteroid treatment which significantly delays disease progression, but this comes at the cost of side effects. Edasalonexent would be a more targeted alternative. Unfortunately, while initial clinical trials were promising, no significant difference was observed between treated and placebo groups for the North Star Ambulatory Assessment or timed function tests in the double-blind placebocontrolled phase 3 clinical trial. We commend the authors for publishing these data so quickly and sharing the information with the community. While this is not the result the field was hoping for, the data from the placebo group can be used to optimize clinical trial design for other studies using the same clinical endpoints.

We encourage you to read these and the other papers in this issue, because we are pleased to present it and are proud of its contents. We encourage other researchers to submit their studies to this or any journal: ours is not the first initiative to promote this [11], and, while we hope one day it will not be necessary to dedicate special issues or have specific calls for sharing these results, we applaud the latest drive of journals reminding researchers that manuscripts such as the ones included here are welcome. As mentioned, the journal will have a special section dedicated to these types of studies, so additional work can be submitted beyond this current issue. Our experience as editors of this issue have calmed our fears: not only have researchers submitted manuscripts of very good standards, reviewers have been happy to collaborate and we expect that readers will also acknowledge this effort citing these manuscripts in the future.

\section{ACKNOWLEDGMENTS}

Both authors are members of COST Action DARTER, which is a funded by the EU (grant code CA1703, October 2018-October 2022), V. AG holds a Miguel Servet Fellowship from the ISCIII (CPII17/00004), part-funded by ERDF/FEDER. V. A-G also acknowledges funding from Ikerbasque (Basque Foundation for Science). AAR is a member of the Netherlands Neuromuscular Center, the Duchenne Centrum Netherlands (funded by Spieren voor Spieren) and the European Reference Network for rare neuromuscular diseases EURO-NMD.

\section{DISCLOSURE STATEMENT(S)}

V.A-G is a shareholder of Miramoon Pharma SL, a company developing neuromuscular treatments, but not related to the focus of this manuscript. 
AAR discloses being employed by LUMC which has patents on exon skipping technology, some of which has been licensed to BioMarin and subsequently sublicensed to Sarepta. As co-inventor of some of these patents AAR is entitled to a share of royalties. AAR further discloses being ad hoc consultant for PTC Therapeutics, Sarepta Therapeutics, CRISPR Therapeutics, Summit PLC, Alpha Anomeric, BioMarin Pharmaceuticals Inc., Eisai, Astra Zeneca, Entrada, Takeda, Splicesense, Santhera, Galapagos, Audentes, Bridge Bio, Global Guidepoint and GLG consultancy, Grunenthal, Wave and BioClinica, having been a member of the Duchenne Network Steering Committee (BioMarin) and being a member of the scientific advisory boards of ProQR, Eisai, hybridize therapeutics, silence therapeutics, Sarepta therapeutics and Philae Pharmaceuticals. Remuneration for these activities is paid to LUMC. LUMC also received speaker honoraria from PTC Therapeutics and BioMarin Pharmaceuticals and funding for contract research from Italpharmaco, Sapreme and Alpha Anomeric. Project funding is received from Sarepta Therapeutics. All authors report no known competing financial interests or personal relationships that could have appeared to influence the work reported in this manuscript.

\section{REFERENCES}

[1] Echevarria L, Malerba A, Arechavala-Gomeza V. Researcher's Perceptions on Publishing "Negative" Results and Open Access. Nucleic Acid Ther. 2020.

[2] Fanelli D. Negative results are disappearing from most disciplines and countries. Scientometrics. 2011;90(3):891-904.

[3] Fanelli D. Do Pressures to Publish Increase Scientists' Bias? An Empirical Support from US States Data. PLoS ONE. 2010;5(4):e10271.
[4] Fanelli D. Opinion: Is science really facing a reproducibility crisis, and do we need it to? Proceedings of the National Academy of Sciences of the United States of America. 2018;115(11):2628-31.

[5] Baker M. 1,500 scientists lift the lid on reproducibility. Nature. 2016;533(7604):452-4.

[6] Yong E. Replication studies: Bad copy. Nature. 2012;485(7398):298-300.

[7] Greco A, Straasheijm KR, Mul K, van den Heuvel A, van der Maarel SM, Joosten LAB, et al. Profiling Serum Antibodies Against Muscle Antigens in Facioscapulohumeral Muscular Dystrophy Finds No Disease-Specific Autoantibodies. Journal of neuromuscular diseases. 2021.

[8] Sinaei F, Fatehi F, Oveis Gharan S, Ehsan S, Kamali K, Amirzargar A, Mahmoudi M, Vaghefifar A, Nafissi S. Association of HLA Class II Alleles with Disease Severity and Treatment Response in Iranian Patients with Myasthenia Gravis. J Neuromuscul Dis. 2021 epub May 26

[9] Vaubourg C, Gicquel E, Richard I, Lostal W, Bellec J: Minimal Consequences of CMAH and DBA/2J Background on a FKRP Deficient Model. J Neuromuscul Dis. 2020 Epub Aug 17.

[10] Finkel RS, McDonald CM, Lee Sweeney H, Finanger E, Neil Knierbein E, Wagner KR, Mathews KD, Marks W, Statland J, Nance J, McMillan HJ, McCullagh G, Tian C, Ryan MM, O'Rourke D, Müller-Felber W, Tulinius M, Bryan Burnette W, Nguyen CT, Vijayakumar K, Johannsen J, Phan HC, Eagle M, MacDougall J, Mancini M, Donovan JM; (For the PolarisDMD Study Group): A Randomized, Double-Blind, Placebo-Controlled, Global Phase 3 Study of Edasalonexent in Pediatric Patients with Duchenne Muscular Dystrophy: Results of the PolarisDMD Trial. J Neuromuscul Dis. 2021 Epub Jun 10

[11] Eilers W, Denti MA. Delivery of Antisense RNA Therapeutics: Turning Negative Results Into a Positive Development. Nucleic Acid Ther. 2021;31(3):183-4. 